\title{
CAUSALITY AND ROBUSTNESS IN THE REMOTE SENSING OF ACOUSTIC PRESSURE, WITH APPLICATION TO LOCAL ACTIVE SOUND CONTROL
}

\author{
S.J. Elliott, W. Jung and J. Cheer
}

Institute of Sound and Vibration Research, University of Southampton, Southampton, U.K., SO17 1BJ

\begin{abstract}
It is often difficult to position microphones at the ear position of listeners to directly monitor the perceived sound, in local active sound control systems for example. The pressure at these positions can be estimated using virtual sensing with an array of remote monitoring microphones, however, if some assumptions are made about the sound field. In active control, the sound field due to the secondary sources can be reasonably easily accounted for but the primary sound field, which is to be controlled, will in general be due to a number of potentially correlated primary sources, whose positions are unknown and may vary in time. The virtual sensing in this application thus needs to be robust to the properties of the primary sound field, both in the choice of the remote monitoring microphone positions and in the design of the filters used to process these to estimate the pressure at the desired position. If the controller is feedforward, the causality of these filters may also be relaxed if the adaptive algorithm is designed to minimise a delayed virtual error signal. This paper describes examples of such robust design, particularly applied to the local active control of road noise in vehicles.
\end{abstract}

Index Terms - Remote microphone method, local active sound control, Robustness

\section{INTRODUCTION}

It may be impossible to place a microphone exactly at the position where the pressure needs to be monitored in various applications, and so remote sensing must be used, typically using an array of monitoring microphones. An example would be local active sound control at the listener's ear in a vehicle [1-8], and a number of methods of remote sensing have been developed for this application [9]. We are particularly interested in the active control of random road noise in vehicles [8], for which time-advanced reference signals are available so that adaptive feedforward control algorithms can be used [10]. To achieve good performance in this application it is important that the latencies in the direct control path

\footnotetext{
This research was jointly funded by an EPSRC industrial CASE studentship (Award no. 14220108) with Jaguar Land Rover (JLR). The authors are especially thankful to Dr Delphine Nourzad and Mr Xavier Vinamata of JLR for their support as industrial supervisors.
}

are kept to a minimum [11]. The error signals derived from the remote sensing, however, are only used to adapt the feedforward controller, and so although the use of a delayed error signal may affect the convergence time, it will not change the steady-state performance of such a controller under stationary conditions. The use of such a delayed error signal is described in section 2 .

The general arrangement of a remote sensing system in this application is shown in Figure 1, in which the monitoring microphones, with outputs $\boldsymbol{d}_{m}$, are assumed to be linearly processed by an observation filter, $\boldsymbol{O}$, to best estimate the signal at the remote virtual microphones. Another important issue in practice is the design of such an observation filter so that it is robust to the details of the stochastic sound field being sensed, which is assumed in Figure 1 to be generated by an array of partially correlated primary noise sources, $\boldsymbol{v}$. The robustness analysis for remote sensing in section 3 constitutes the main contribution of this paper and section 4 provides an example of its application.

\section{ADAPTATION WITH A DELAYED, REMOTELY-SENSED, ERROR SIGNAL}

Figure 2 shows the block diagram of an adaptive feedforward controller using the filtered reference LMS algorithm and the "remote microphone technique" (RMT) [9, 12], incorporating a modelling delay of $\Delta$ samples in the observation filter $[8,13]$. The modelling delay also has to be included in the estimation of the response from the secondary source to the virtual error microphone, $\hat{\boldsymbol{G}}_{e}$, both in the remote microphone arrangement, coloured yellow in Figure 2, and in the internal model of this, used to generate the filtered reference signals for the adaptive LMS algorithm. Figure 3 shows a comparison of the results of using an adaptive feedforward algorithm to implement a local active controller on a headrest in the laboratory, with either the standard RMT or the delayed RMT, when a single loudspeaker, used as the primary source, was placed in front of the headrest [7]. In this case the error microphone is closer to the primary source than the monitoring microphones, so the ideal observation filter is non-causal and the delayed RMT allows considerably better attenuation of the disturbance than the stand. This is because the delayed RMT essentially allows a non-causal observation filter, which 


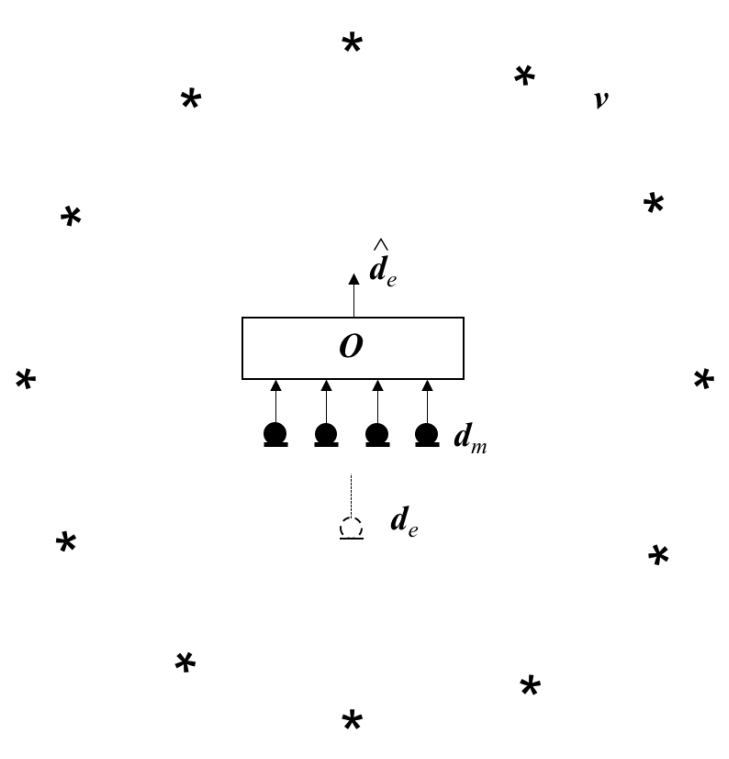

Fig. 1. Remote sensing arrangement in which the outputs of an array of monitoring microphones, $\boldsymbol{d}_{m}$, is processed by an observation filter, $\boldsymbol{O}$, to give an estimate, $\hat{\boldsymbol{d}}_{e}$, of the output of a remote virtual microphone, $\boldsymbol{d}_{e}$. The stochastic signals at the microphones are assumed to be generated by an array of partially correlated primary sources, $\boldsymbol{v}$, as indicated by stars.

estimates the virtual error signals from the physical monitoring microphone signals. In other experiments, when the loudspeaker was positioned behind the headrest, the ideal observation filter was causal and then there is little difference between the performance of the standard and delayed RMT implementations.

It should be emphasised that although the adaptation of the controller, $\boldsymbol{W}$, is a real-time operation, the design of the observation filter must generally be performed in an identification phase prior to implementation, since it requires access to the signals from the virtual microphones, which are removed in real-time operation. If FIR filters are used to implement the observation filter, time domain methods may be used for its design $[8,14]$. With a reasonable modelling delay, however, the time domain filters are well approximated by the Fourier transforms of the filters designed in the frequency domain. Frequency domain design can give considerable insight into the robust design of these observation filters, as considered below.

\section{ROBUST DESIGN IN REMOTE SENSING}

Considering the stochastic signals in a single frequency bin, the estimation error between the actual pressures at the virtual microphones and their remote estimate using the observation filter can be written as

$$
\boldsymbol{\epsilon}=\boldsymbol{d}_{e}-\hat{\boldsymbol{d}}_{e}=\boldsymbol{d}_{e}-\boldsymbol{O} \boldsymbol{d}_{m}
$$

where the dependence on frequency has been suppressed for notational convenience. Assuming that $\boldsymbol{d}_{m}$ and $\boldsymbol{d}_{e}$ are generated by the array of primary sources in Figure 1, the estimation error can be written as

$$
\boldsymbol{\epsilon}=\boldsymbol{P}_{e} \boldsymbol{v}-\boldsymbol{O} \boldsymbol{P}_{m} \boldsymbol{v}
$$

where $\boldsymbol{P}_{e}$ and $\boldsymbol{P}_{m}$ are the matrices of responses from the array of primary sources to the vectors of virtual and monitoring microphones, as shown in Figure 2. For a robust design we assume that these two response matrices are subject to uncertainty, due for example to perturbations in the acoustic field, so that

$$
\begin{gathered}
\boldsymbol{P}_{e}=\boldsymbol{P}_{e_{0}}+\boldsymbol{\Delta} \boldsymbol{P}_{e}, \\
\boldsymbol{P}_{m}=\boldsymbol{P}_{m_{0}}+\boldsymbol{\Delta} \boldsymbol{P}_{m},
\end{gathered}
$$

where $\boldsymbol{P}_{e_{0}}$ and $\boldsymbol{P}_{m_{0}}$ are the nominal responses and $\boldsymbol{\Delta} \boldsymbol{P}_{e}$ and $\boldsymbol{\Delta} \boldsymbol{P}_{m}$ are perturbations away from these.

We treat the elements of $\Delta \boldsymbol{P}_{e}$ and $\Delta \boldsymbol{P}_{m}$ as random perturbations [15], which are uncorrelated with the actual responses and also between themselves so that

$$
\begin{array}{r}
<\boldsymbol{P}_{m_{0}}^{H} \boldsymbol{\Delta} \boldsymbol{P}_{m}>=0 \\
<\boldsymbol{P}_{e_{0}}^{H} \boldsymbol{\Delta} \boldsymbol{P}_{m}>=0 \\
<\boldsymbol{\Delta} \boldsymbol{P}_{e} \boldsymbol{\Delta} \boldsymbol{P}_{m}>=0
\end{array}
$$

where $\langle>$ denotes averaging over an ensemble of different perturbations, and a similar set of conditions applied to $\Delta \boldsymbol{P}_{e}$. A robust observation filter can now be designed using the methods discussed in $[10,15]$, to minimise the expectation value of the sum of the mean square errors which is

$$
\boldsymbol{O}_{o p t}=<\boldsymbol{P}_{e} \boldsymbol{S}_{v v} \boldsymbol{P}_{m}^{H}>\left[<\boldsymbol{P}_{m} \boldsymbol{S}_{v v} \boldsymbol{P}_{m}^{H}>\right]^{-1}
$$

where $\boldsymbol{S}_{v v}$ is the power spectral density matrix of the primary sources. Using the assumed properties of the perturbed responses described above, this reduces to

$$
\boldsymbol{O}_{o p t}=\boldsymbol{P}_{e_{0}} \boldsymbol{S}_{v v} \boldsymbol{P}_{m_{0}}^{H}\left[\boldsymbol{P}_{m_{0}} \boldsymbol{S}_{v v} \boldsymbol{P}_{m_{0}}^{H}+\boldsymbol{B}\right]^{-1}
$$

where

$$
\boldsymbol{B}=<\Delta \boldsymbol{P}_{m} \boldsymbol{S}_{v v} \boldsymbol{\Delta} \boldsymbol{P}_{m}^{H}>\approx \beta \boldsymbol{I}
$$

If the perturbations in the elements of $\boldsymbol{P}_{m}$ and the primary sources are reasonably well uncorrelated but have similar mean square values, then the matrix $\boldsymbol{B}$ will approximate a diagonal matrix and can be written as $\beta \boldsymbol{I}$, where $\beta$ is a scalar regularisation parameter. Thus the robust design of the observation filter used for remote sensing has exactly the same Tikhonov-regularised form as that which penalises the magnitude of the elements in the observation filter [6]. Also, if there 


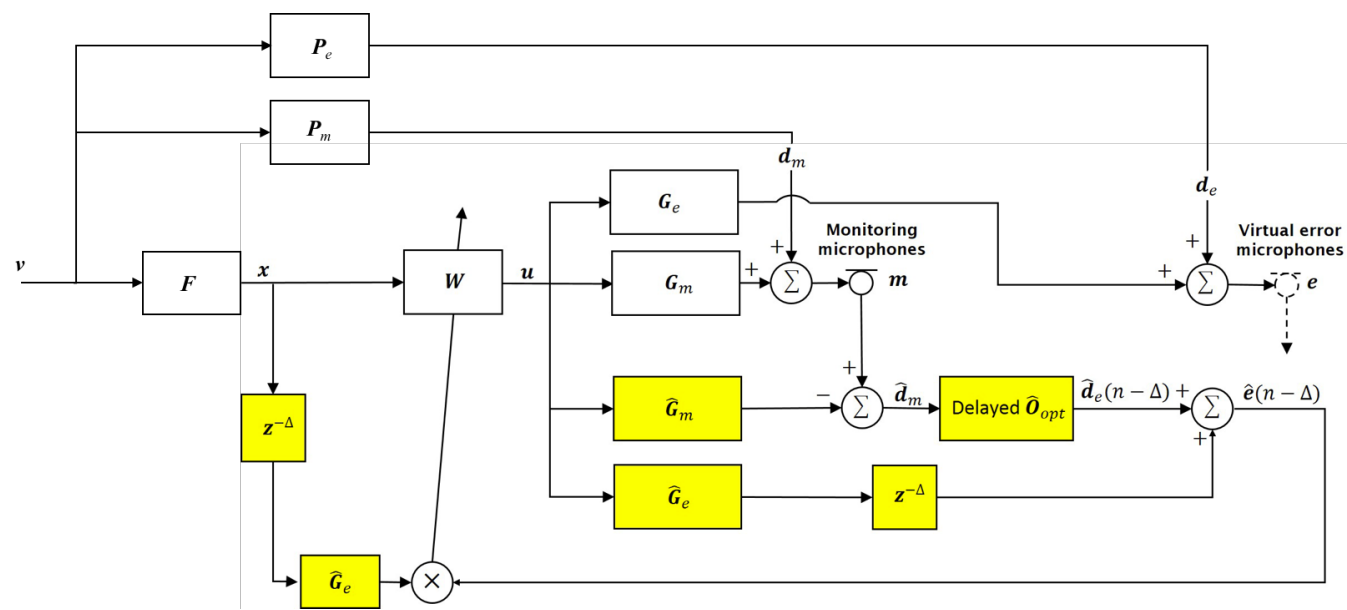

Fig. 2. Block diagram of the filtered-reference LMS algorithm for adaptive feedforward control in which the error signal is derived using the remote microphone method, in yellow, with a modelling delay, $\Delta$.

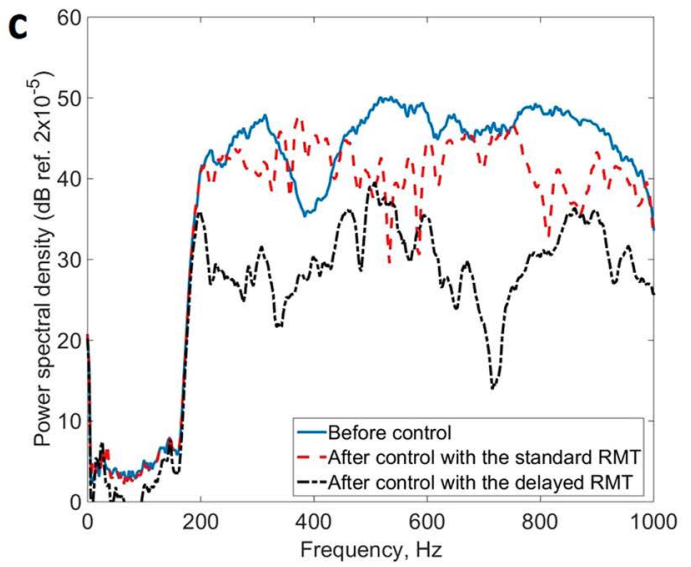

Fig. 3. Power spectral density of the signal measured at the error microphone without control (blue solid line), with control using the standard RMT (red dashed line) and with control using the delayed RMT (blue dotted line).

are components of the primary noise field that couple into the monitoring microphones more efficiently than into the error microphones, these will act as sensor noise in the design of the observation filter, further regularising the matrix being inverted in its design [16].

Considerable insight into the effect of the regularisation parameter, $\beta$, can be obtained by considering the variation of the condition number of the matrix being inverted in equation (9) with this parameter, and the magnitude of the observation error, as plotted in Figure 4. This has been generated for the arrangement shown in Figure 1 in the case of a diffuse sound field, for which there are a large number of uniformlydistributed and uncorrelated primary sources, in which case the spatial cross-correlation functions in eq. (9) have an ana- lytical formulation $[6,17]$. The separation between the monitoring microphones in Figure 1 was $0.1 \mathrm{~m}$ at a frequency of about $135 \mathrm{~Hz}$ in this simulation, so that the separation between the microphones is about $4 \%$ of the acoustic wavelength, and the virtual microphone was at a distance of $0.2 \mathrm{~m}$ in front of the array. Clearly there is a range of values of the regularisation parameter for which the observation error is not significantly increased, but the condition number of the matrix inverted in eq. (9), which is a measure of the sensitivity of the filter to uncertainties in the data, is considerably reduced. Choosing the regularisation factor to be about $10^{4}$, for example, gives a condition number which is about 200, instead of about $2 \times 10^{6}$ if the regularisation factor is very small, and an estimation error which is within $0.05 \mathrm{~dB}$ of the best that can be achieved, with little regularisation, which is about $-11 \mathrm{~dB}$ in this case.

\section{EXAMPLES OF ROBUST REMOTE SENSING IN A VEHICLE}

Jung [8] describes a series of experiments in which the pressure waveforms due to the road noise in a large SUV were measured at 16 remote monitoring microphones and at the ear positions of a dummy head, as shown in Figure 5, under different road conditions. Different observation filters were designed, based on these different road conditions, and the dashed line in Figure 6 shows the normalised estimation error at the left ear of the dummy head as a function of frequency, as calculated from the data when the vehicle was driven over a smooth road and using an observation filter calculated from the same data. The estimation error increases with frequency, but is less than $-10 \mathrm{~dB}$ below about $400 \mathrm{~Hz}$. Also shown, as the solid line, in this Figure, is the estimation error calculated from the data on the smooth road but using an observation filter calculated from the data when the vehicle was driven 


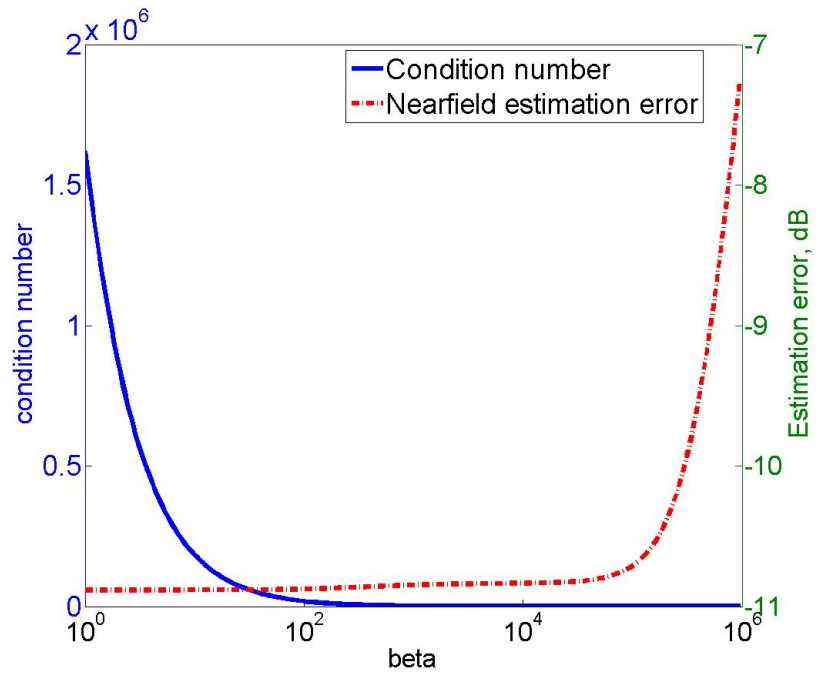

Fig. 4. Variation with the regularisation parameter, $\beta$, of the condition number of the matrix that is inverted in the calculation of the optimum observation filter, solid line, and the level of the normalised estimation error, dashed line.

over a rough road. It can be seen that although the estimation error is somewhat larger at a few frequencies, it is generally of a similar level to that obtained with the observation filter designed for the smooth road conditions. The estimation error gets progressively larger if less than 16 monitoring microphones are used for remote sensing, even when an exhaustive search was used to find the best sub-set [8]. It is also possible to use an additional $l 1$ constraint in the design of the observation filter to more easily select the best subset of monitoring microphones using a LASSO-type algorithm, as described for example by [18].

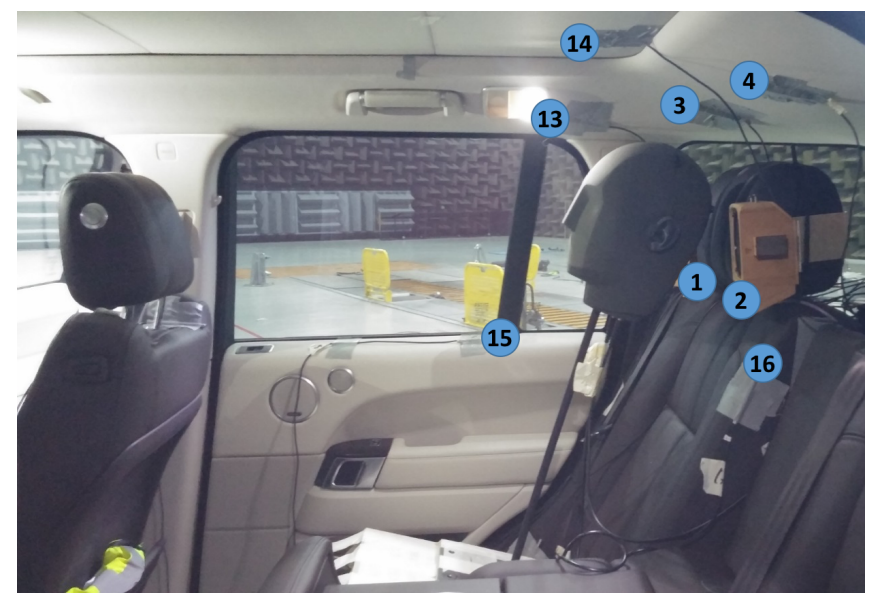

Fig. 5. The arrangement of monitoring microphones and the dummy head used to record the pressure waveforms due to the road noise in a large SUV when driven over different road surfaces.

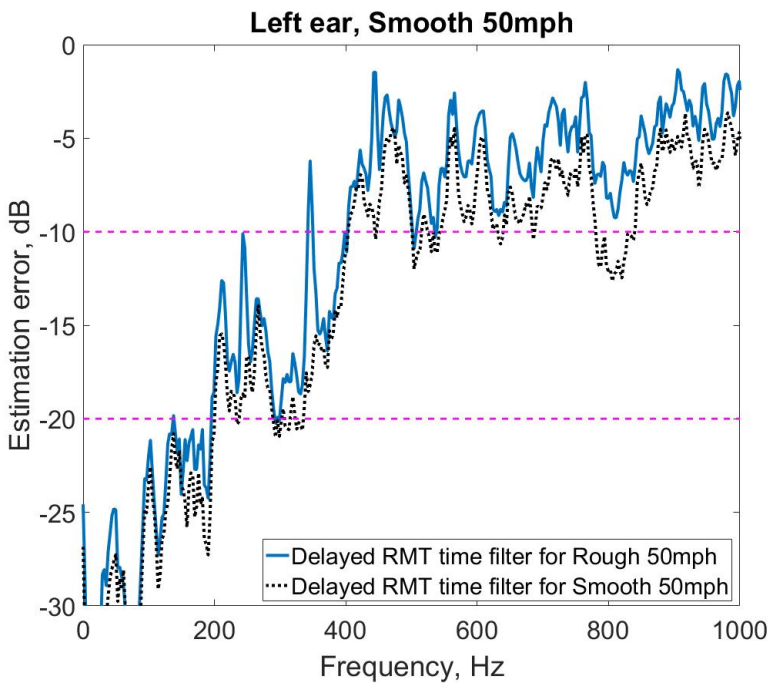

Fig. 6. The normalised estimation error at the left ear of the dummy head as a function of frequency, calculated using the delayed remote microphone technique (RMT), when the vehicle was driven over a smooth road. The dashed line shows the results when the observation filter was calculated using the same data, for the smooth road, and the solid line shows the error on the smooth road when the observation filter was calculated using data from a rough road.

\section{CONCLUSIONS}

It has been shown that although causality can be very important in designing the control filters for an adaptive feedforward controller, there is less of a causality issue for the remote sensing of an error signal that is only used to adapt the controller. The same conclusion would not hold if a feedback control was used instead however, since the error signal is then effectively also used as a reference signal in such an arrangement $[10,19,20]$. The relaxed causality constraint allows frequency domain design of the filter used for the remote sensing. By minimising the mean square estimation error over an ensemble of different primary fields, it is shown that the robust design of these filters is equivalent to calculating it using Tikhonov regularisation. This is illustrated by an example using data measured in a vehicle under two different road noise conditions. 


\section{REFERENCES}

[1] H. F. Olson and E.G. May, "Electronic sound absorber," The Journal of the Acoustical Society of America, vol. 25, no. 6, pp. 1130-1136, 1953.

[2] J Garcia-Bonito, SJ Elliott, and CC Boucher, "Generation of zones of quiet using a virtual microphone arrangement," The journal of the Acoustical Society of America, vol. 101, no. 6, pp. 3498-3516, 1997.

[3] M. Pawelczyk, "Adaptive noise control algorithms for active headrest system," Control Engineering Practice, vol. 12, no. 9, pp. 1101-1112, 2004.

[4] P.S. Booji and A.P. Berkhoff, "Virtual sensors for local, three dimensional, broadband multiple-channel active noise control and the effects on the quiet zones," Proceedings of ISMA2010 including USD2010, pp. 151166, 2010.

[5] W. Jung, S.J. Elliott, and J. Cheer, "Combining the remote microphone technique with head-tracking for local active sound control," The Journal of the Acoustical Society of America, vol. 142, no. 1, pp. 298-307, 2017.

[6] W. Jung, S.J. Elliott, and J. Cheer, "Estimation of the pressure at a listener's ears in an active headrest system using the remote microphone technique," The Journal of the Acoustical Society of America, vol. 143, no. 5, pp. 2858-2869, 2018.

[7] S.J. Elliott, W. Jung, and J. Cheer, "Head tracking extends local active control of broadband sound to higher frequencies," Scientific reports, vol. 8, no. 1, pp. 5403, 2018.

[8] W. Jung, S.J. Elliott, and J. Cheer, "Local active control of road noise inside a vehicle," Mechanical Systems and Signal Processing, 2019.

[9] D. Moreau, B. Cazzolato, A. Zander, and C. Petersen, "A review of virtual sensing algorithms for active noise control," Algorithms, vol. 1, no. 2, pp. 69-99, 2008.

[10] S. J. Elliott, Signal Processing for Active Control, Academic Press, London, 2001.

[11] T. J. Sutton, S. J. Elliott, M. A. McDonald, and T. J. Saunders, "Active control of road noise inside vehicles," Journal of Noise Control Engineering, vol. 42, no. 4, pp. 137-146, Jul-Aug 1994.

[12] A. Roure and A. Albarrazin, "The remote microphone technique for active noise control," in INTER-NOISE and NOISE-CON Congress and Conference Proceedings. Institute of Noise Control Engineering, 1999, vol. 1999, pp. 1233-1244.
[13] D. Treyer, S. Gaulocher, S. Germann, and E. Curiger, "Towards the implementation of the noise-cancelling office chair: Algorithms and practical aspects," in Proceedings of of 23rd International Congress on Sound and Vibration, 2016.

[14] S.J. Elliott and J. Cheer, "Modeling local active sound control with remote sensors in spatially random pressure fields," The Journal of the acoustical Society of america, vol. 137, no. 4, pp. 1936-1946, 2015.

[15] S.J. Elliott, J. Cheer, J.-W. Choi, and Y. Kim, "Robustness and regularization of personal audio systems," IEEE Transactions on Audio, Speech, and Language Processing, vol. 20, no. 7, pp. 2123-2133, 2012.

[16] B. Widrow and S. D. Stearns, "Adaptive signal processing," Englewood Cliffs, NJ, Prentice-Hall, Inc, vol. 1, pp. 491, 1985.

[17] D. J Moreau, J. Ghan, BS Cazzolato, and AC Zander, "Active noise control in a pure tone diffuse sound field using virtual sensing," The Journal of the acoustical Society of america, vol. 125, no. 6, pp. 3742-3755, 2009.

[18] M. Adnadjevic, B. Goossens, and D. Botteldooren, “On the array configuration and accuracy of remote in-ear level sensing for in-vehicle noise control applications," Applied Acoustics, vol. 129, pp. 229-238, 2018.

[19] S. J. Elliott and T. Sutton, "Performance of feedforward and feedback systems for active control," IEEE Transactions on Speech and Audio Processing, vol. 4, no. 3, pp. 214-223, May 1996.

[20] J. Cheer and S.J. Elliott, "Multichannel control systems for the attenuation of interior road noise in vehicles," Mechanical Systems and Signal Processing, vol. 60-61, pp. $753-769,2015$. 